\title{
Maxillary Dentigerous Cyst in Association with an Impacted Mesiodens in a Paediatric Patient Treated By Enucleation: A Case Report
}

\author{
Dr. Soumithran C.S ${ }^{1}$, Dr. Arul Dev D.P ${ }^{2}$, Dr. Manu Mathew ${ }^{3,}$ \\ Dr. Manjusha $\mathrm{P}^{4}$, Dr. Nimmi $\mathrm{M}^{5}$

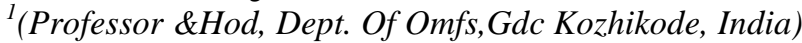 \\ 2 (Junior Resident, Dept. Of Omfs, Gdc Kozhikode, India) \\ 3 (Junior Resident, Dept. Of Omfs, Gdc Kozhikode, India) \\ ${ }^{4}$ (Senior Resident, Dept. Of Oral Pathology, Gdc Kozhikode, India) \\ ${ }_{5}^{5}$ (Junior Resident, Dept. Of Omfs, Gdc Kozhikode, India)
}

\begin{abstract}
Dentigerous cysts are developmental odontogenic cysts and are always associated with an unerupted or developing tooth bud. Mesiodens is a supernumerary tooth found in the anterior maxilla. Supernumerary teeth seldom associate with dentigerous cysts and dentigerous cysts around supernumerary teeth account for $5 \%$ of all dentigerous cysts. Here we report a case of dentigerous cyst in association with a mesiodens in an 8 year old boy. The cyst was treated by enucleation with minimal tissue trauma and nerve preservation.
\end{abstract}

Keywords: Dentegerous cyst, supernumerary teeth, nerve, enucleation

\section{Introduction}

Dentigerous cysts are the most common developmental, odontogenic cysts arising from a crown of impacted, embedded, or unerupted teeth. They are most commonly associated with the crowns of permanent teeth, though few rare cases are reported in association with the crowns of deciduous teeth, complex odontoma, and supernumerary teeth[1]. These cysts are caused by expansion of dental follicles resulting from accumulation of fluid between the tooth crown and epithelial components.[2]The commonest teeth involved are mandibular third molars followed by maxillary canines and the mandibular premolars. It may occur at any age group but the greatest incidence is in second and third decade. They account for about $24 \%$ of all true cysts in the jaws[3]. Supernumerary teeth are extra or accessory teeth that are believed to result from the duplication of either the primary or permanent dentition. Mesiodens is a supernumerary tooth found in the anterior maxilla. Supernumerary teeth seldom associate with dentigerous cysts and dentigerous cysts around supernumerary teeth account for $5 \%$ of all dentigerous cysts[4]. Here we report a case of dentigerous cyst in association with a mesiodens in an 8 year old boy.

\section{Case Report}

An 8 year old male patient reported to the Dept of Oral \& Maxillofacial Surgery with a chief complaint of pain and swelling in the maxillary anterior region for 1 month. The swelling had only slightly increased in size since then. There was no associated history of trauma and the medical history was non contributory. Intraoral examination revealed that maxillary permanent incisors had erupted. However they were malaligned. A diffuse swelling of size $1 \times 1 \mathrm{cms}$ was noticed involving the labial sulcus. The swelling obliterated the labial mucobuccal fold superiorly and inferiorly it extended to the region of the alveolar mucosa. The overlying mucosa was intact and of the same color. No surface discharge was seen. The swelling was nontender and firm in consistency. The maxillary anterior teeth responded positively to vitality tests. A provisional diagnosis of nasolabial cyst was made.

Radiographs were advised. A panoramic radiograph revealed the presence of a supernumerary teeth in the maxillary anterior region. A maxillary occlusal radiograph showed the presence of a well defined unilocular radiolucent lesion surrounding the supernumerary tooth [fig 1].Fine needle aspiration yielded a pale yellow cystic fluid[fig2]. An incisional biopsy was done under local anaesthesia .Histological examination revealed a cystic lining composed of 2-4 cell layered stratified squamous epithelium. The supporting connective tissue capsule was loosely collagenous resemebling primitive ectomesenchyme. Correlating clinically radiographically and histopathologically a final diagnosis of dentigerous cyst was made.

Enucleation was planned under local anesthesia. Patient was anesthetised by giving bilateral infraorbital and nasopalatine nerve blocks. A crevicular incision was placed from right central incisor to left first premolar region with a releasing incision distal to it including the papilla. A mucoperiostel flap was carefully 
raised preserving the marginal gingiva. The bone underneath was studied. Using a bone ronger forceps the thinned out bone overlying the cyst was removed and access to the cyst made[fig3].The cyst lining was analysed and using a periosteal elevator the cyst lining was carefully raised[6].The nasopalatine nerve was identified and was carefully dissected away from the cyst lining. [fig 4]Then the cyst was completely enucleated in toto. Thus the nerve was preserved. The mucoperiostel flap was replaced and sutured with 4-0 silk[fig5].

\section{Discussion}

Dentigerous cysts are developmental odontogenic cysts and are always associated with an unerupted or developing tooth bud .They are the second most common cystic lesion of the jaws, after radicular cysts[5]. The literal meaning of dentigerous is 'tooth bearing'. The dentigerous cysts are epithelial lined sacs that develop from the enamel organ in association with the crown of an unerupted tooth. They are commonly seen associated with impacted mandibular third molars.

Supernumerary teeth can resemble normal teeth, have conical crowns, or bear little resemblance to a normal tooth. Mesiodens is a supernumerary tooth that occur near the midline of the maxilla, almost always posterior to the normal central incisors. Most mesiodentes are conical, as in our case. The prevalence of the mesiodens appears to be to be in the range of $0.15 \%$ to $2.2 \%$ of the population[4]. The mesiodens can erupt or remain impacted and often cause developmental and eruption disturbances of permanent teeth which are adjacent to them, leading to crowding, displacement, diastema and in rare cases, radicular resorption and dentigerous cyst formation. Dentigerous cysts most commonly occur in the 2nd and 3rd decades of life and show a male predilection[2]. In the current case the lesion was reported in an 8 year old male patient who had severely malaligned anteriors.

The exact histogenesis of the dentigerous cyst is not known. It is stated that the dentigerous cyst develops around the crown of an unerupted tooth by accumulation of fluid either between the reduced enamel epithelium and enamel or in between the layers of the enamel organ. [fig 6]This fluid accumulation occurs as a result of the pressure exerted by an erupting tooth on an impacted follicle, which obstructs the venous outflow and thereby induces rapid transudation of serum across the capillary wall[3].

A broad range of conditions may lead to a clinical presentation of painless swelling along the upper lip. Differential diagnosis of median palatine cyst, nasopalatine duct cyst, radicular cyst, odontogenic keratocyst (OKC) or adenomatoid odontogenic tumor (AOT) was considered. The characteristic feature of the lesion involving an unerupted tooth crown in the anterior region of the maxilla along with the radiographical and histological examination confirmed the diagnosis of dentigerous cyst in the current case.

Radiographically, the dentigerous cyst typically appears as a well-circumscribed, unilocular, usually symmetric radiolucency around the crown of an impacted tooth. An important diagnostic point is that this cyst attaches at the cementoenamel junction. Three types of dentigerous cyst have been described radiographically: (a) The central variety, in which the tooth crown is enclosed by the radiolucency, and the crown protrudes into the cystic lumen. (b) The lateral variety in which the cyst occurs laterally along the tooth root, thus, partially surrounding the crown. (c) The circumferential variety exists when the cyst not only surrounds the crown, but also extends down along the root surface, thus, giving the impression of the tooth within the cyst[6]. CT scan provides superior bony detail, size and extent of the lesion with determination of orbital or nasal invasion or involvement. Therefore, CT may be more valuable than plain radiographs[4].

Management of a supernumerary tooth depends on the type and position of the tooth and effect on the adjacent teeth. Removal of the supernumerary has been recommended where there is evidence of the associated pathology, eruption disturbances, displacement of the incisors or where the spontaneous eruption of the supernumerary tooth has occurred. Marsupialization has been recommended for dentigerous cysts in children to allow the eruption of unerupted tooth; and should be preferred for large cysts where enucleation and tooth removal might result in damage to the nerve and blood vessels supplying the adjacent teeth[4]. When the supernumerary tooth is evident, the direction of the crown, the location, the influence on adjacent teeth, the resorption of adjacent roots and the formation of dentigerous cysts should be carefully evaluated.

\section{Conclusion}

There are various treatment modalities for cyst enucleation. But the success of the treatment depends on the complete removal of the cyst lining and elimination of the pathology with the most atraumatic method as possible. Thus, preserving the nerve and its content will improve the patient compliance and least post operative disturbances as such . 


\section{Figures}

Fig 1:

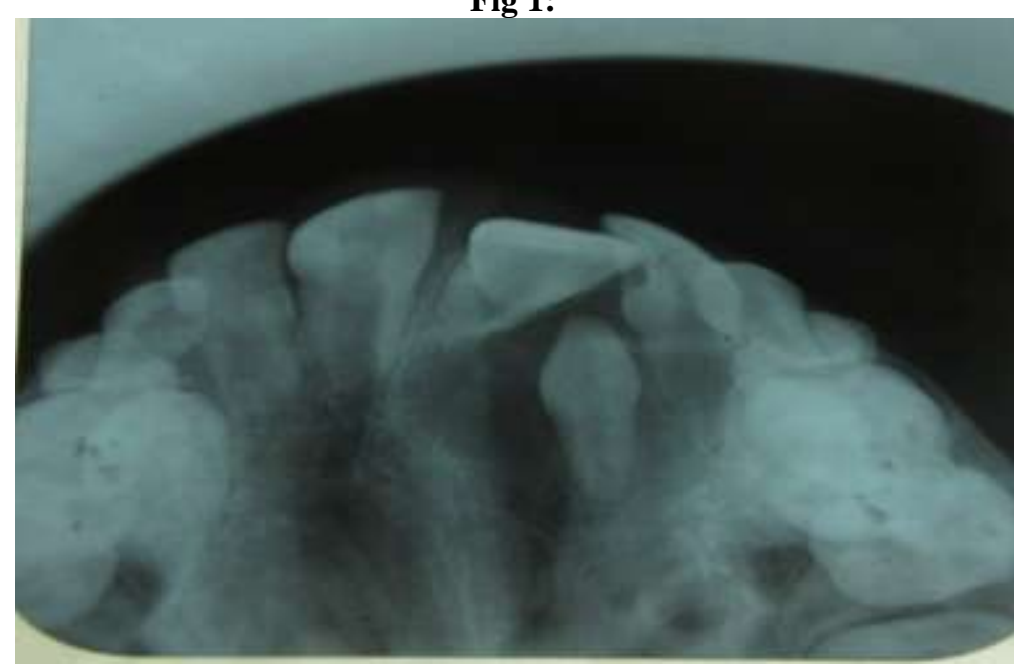

occlusal view showing mesiodens with cyst

FIg 2

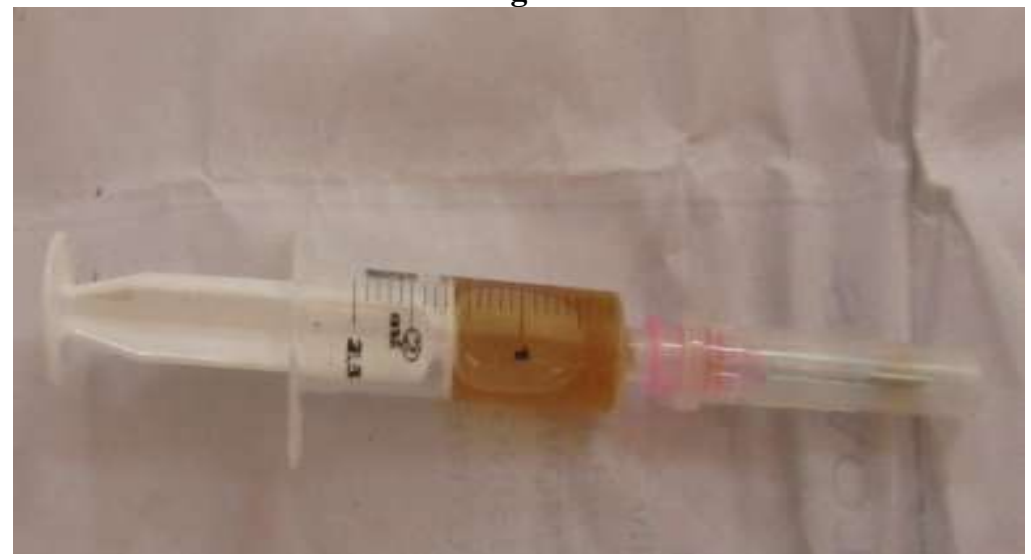

cyst aspirate with straw coloured fluid with cholosterol crystals

Fig 3:

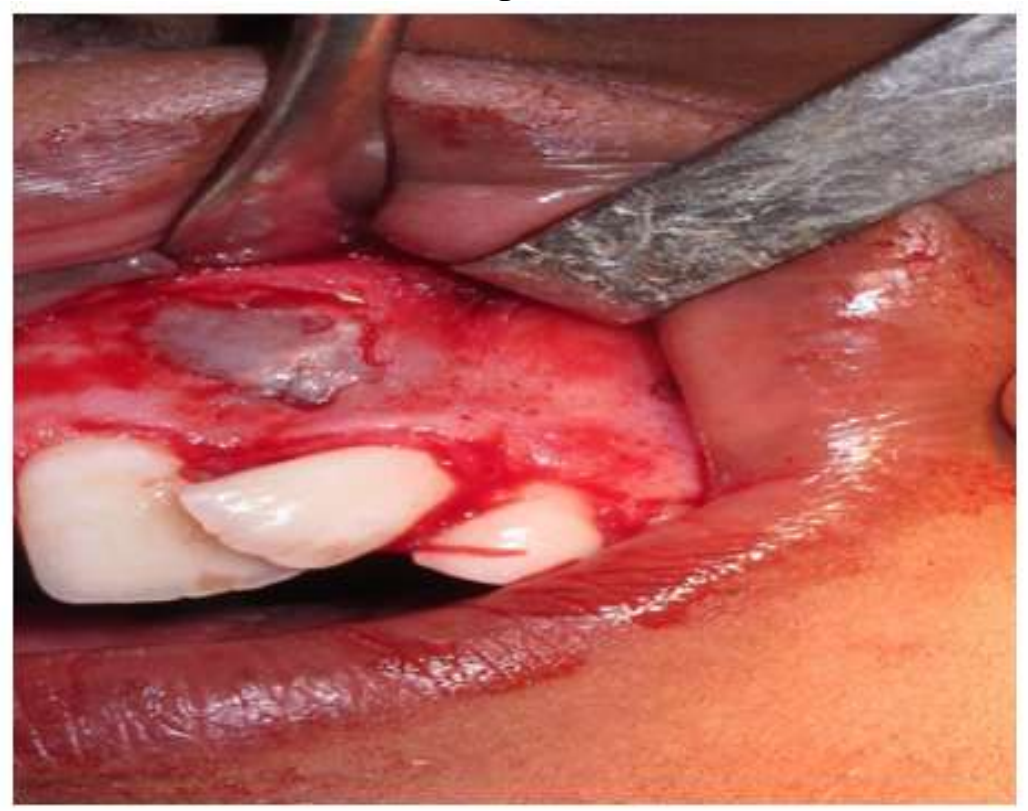


mucoperiosteal flap raised and cyst lining identified.

Fig 4

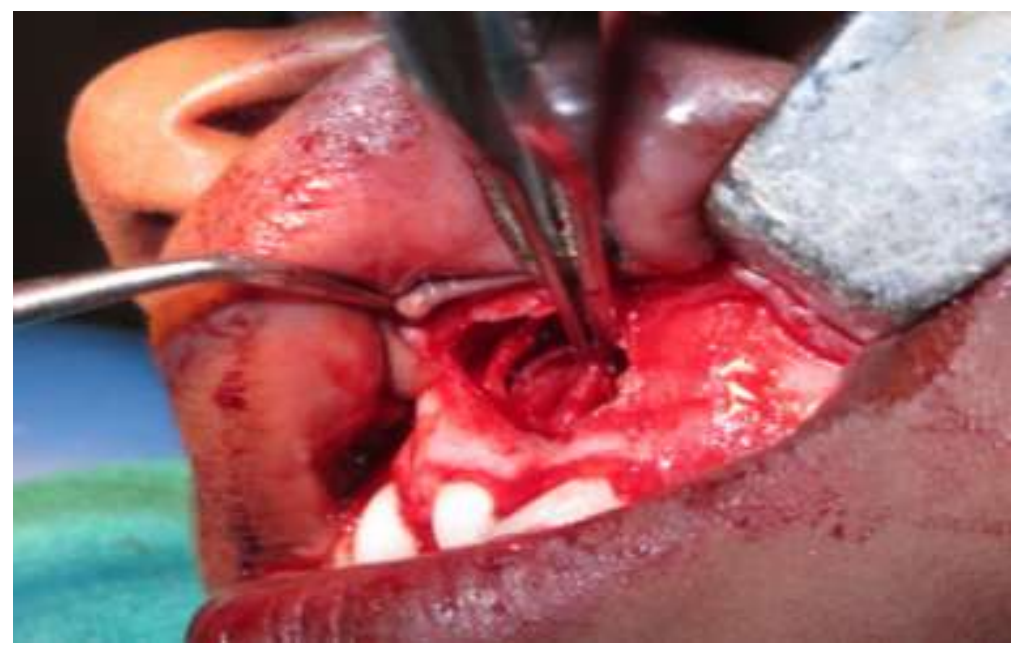

cyst enucleation with nerve preservation

fig 5

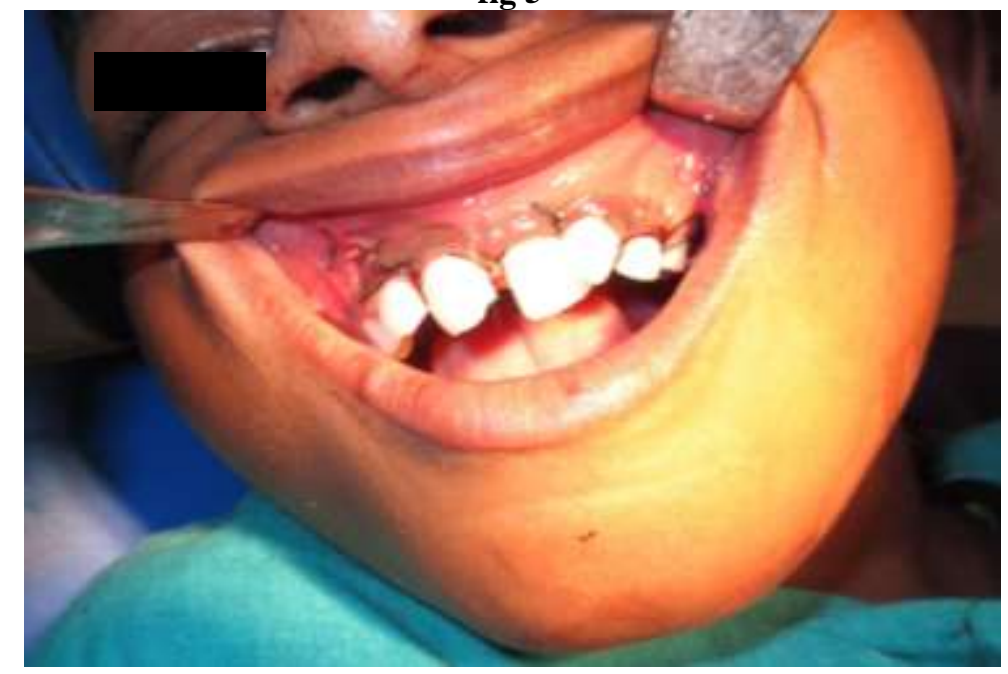

suturing of surgical wound using 4-0 silk

fig 6 


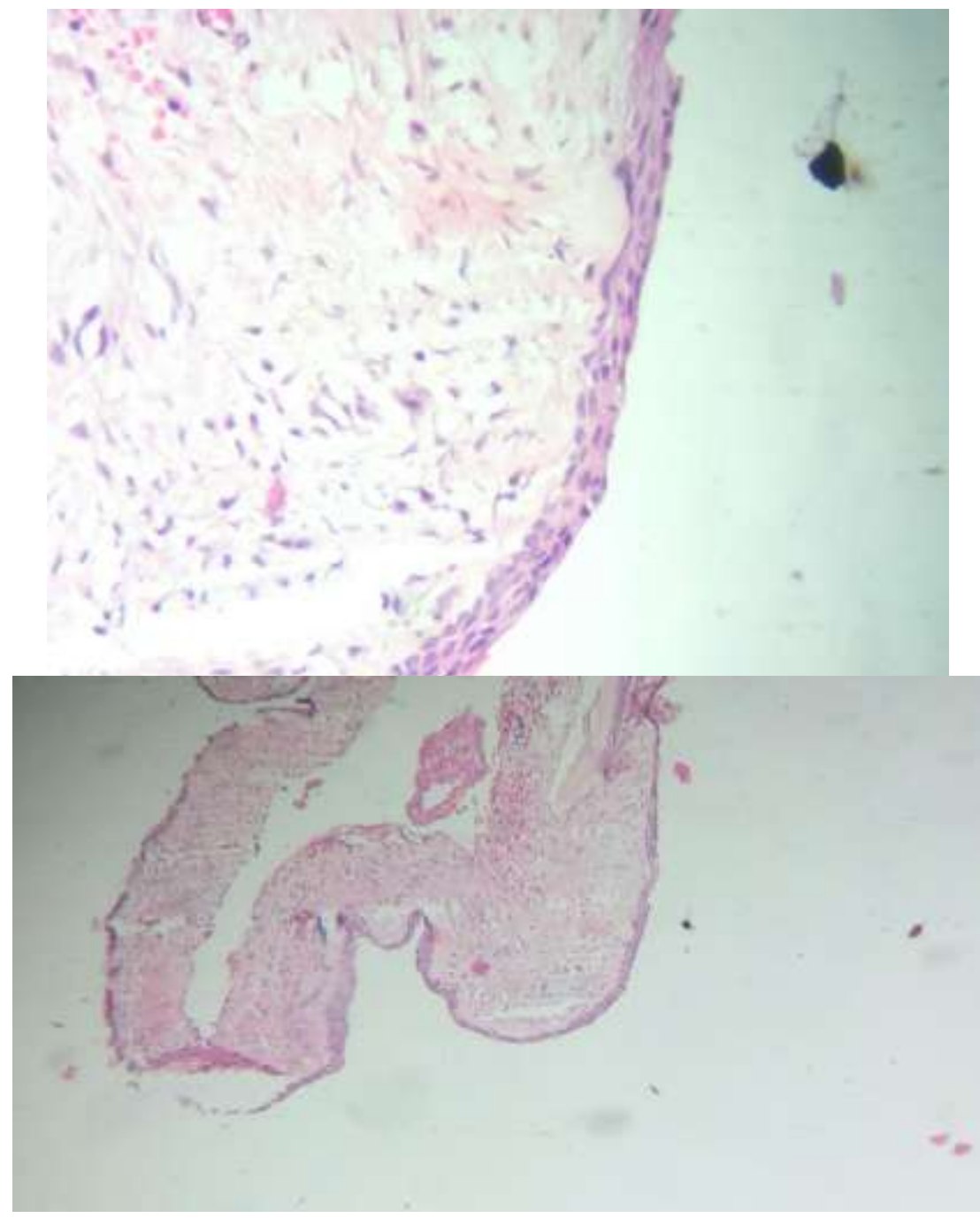

histopathology showing features of dentegerous cyst

\section{References}

[1]. Kasat VO, Karjodkar FR, Laddha RS. Dentigerous cyst associated with an ectopic third molar in the maxillary sinus: A case report and review of literature. Contemp Clin Dent 2012;3:373 6.

[2]. Kalaskar R R, Tiku A, Damle S G. Dentigerous cysts of anterior maxilla in a young child: A case report. J Indian Soc Pedod Prev Dent [serial online] 2007 [cited 2016 Mar 20 ];25:187ᄀ190

[3]. Naveen Kumar. M, Rama Devi. S, Shreenivas Vanaki. S, Puranik. R.S. Dentigerous Cyst Occurring in Maxilla Associated with Supernumerary Tooth Showing Cholesterol Clefts-A Case Report. International Journal Of Dental Clinics Volume 2 Issue 2 AprilJune 2010

[4]. John T, Guna Shekhar M , Koshy M. Dentigerous cyst associated with supernumerary teeth: a report of three cases.JCDR 2010.june vol : 4 issue : 3 page : $2601 \neg 2606$

[5]. Deepak JHH, Sana RM, Vinothini G, Thanigainathan R, Kandasamy M. Maxillary Dentigerous Cyst With Impacted Mesiodens - A Case Report. J Adv Med Dent Scie Res 2015;3(3):119-122

[6]. Shamimul Hasan, Syed Ansar Ahmed, L. Bhaskar Reddy. Dentigerous cyst in association with impacted inverted mesiodens:Report of a rare case with a brief review of literature. Int J Appl Basic Med Res. 2014 Sep; 4(Suppl 1): S61-S64. 